\title{
Asymmetric properties of heart rate microstructure
}

\author{
Przemyslaw Guzik \\ Department of Cardiology-Intensive Therapy and Internal \\ Diseases, Poznan University of Medical Sciences, Poland \\ (D) http://orcid.org/0000-0001-9052-5027 \\ Corresponding author: pguzik@ptkardio.pl \\ Jarosław Piskorski \\ Institute of Physics, University of Zielona \\ Góra, Zielona Góra, Poland \\ (iD) https://orcid.org/0000-0002-0866-734X
}

DOI: https://doi.org/10.20883/medical.e436

\author{
Keywords: cardiovascular time series, heart \\ rate asymmetry, heart rate variability, heart rate \\ microstructure, risk prediction
}

Published: 2020-06-30

How to cite: Guzik P, Piskorski J. Asymmetric properties of heart rate microstructure. JMS [Internet]. 2020 Jun 30;89(2):e436. doi:10.20883/medical.e436

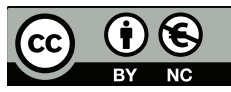

(C) 2020 by the author(s). This is an open access article distributed under the terms and conditions of the Creative Commons Attribution (CC BY-NC) licencse. Published by Poznan University of Medical Sciences

\begin{abstract}
The duration of each cardiac cycle is measured on an ECG as the distance between the peaks of consecutive $R$ waves (RR interval), with the inverse value corresponding to the heart rate (HR) changing in a beat-to-beat manner. HR accelerations are reflected as shortenings of the RR intervals, HR decelerations as the lengthening of RR intervals. HR asymmetry is a physiological phenomenon caused by an unequal input of HR decelerations and accelerations to the HR variability. Naturally occurring consecutive values of RR intervals create a time series composed of acceleration and deceleration runs of differing lengths. For example, a single HR acceleration, a pair of HR decelerations, a run consisting of five consecutive HR decelerations or a run composed of eight accelerations in a row. These runs make up the so-called heart rate microstructure that has asymmetric properties due to the unequal contribution of acceleration and deceleration runs. The asymmetry of the HR microstructure is physiological in healthy individuals, however, the asymmetric properties can be significantly altered in some clinical conditions, such as myocardial infarction, obstructive sleep apnoea, chronic obturatory pulmonary disease or sepsis in infants. An abnormal HR microstructure has predictive value in survivors of myocardial infarction or patients with clinical indications for exercise treadmill stress test, e.g., for total mortality. In this review, we present and explain how the asymmetric properties of HR microstructure can be quantified, summarising the available data regarding the clinical and predictive value of this phenomenon and its analysis.
\end{abstract}

The sinus node is the only physiological pacemaker of the human heart and its electrical depolarisations are the major but not the sole determinant of the length of each cardiac cycle [1-11]. In a normal ECG, the direct activity of the sinus node is practically invisible, but its indirect effects on the atria are observed as the $P$ wave (Figure 1). After the right and left atria are depolarised, the electrical potential is conducted through the atrial node, His Bundle system and the Purkinje fibres finally reaching the myocardial cells of both ventricles. Each QRS complex in ECG proves that the right and left ventricles have been depolarised $[1,3,7,11]$. The duration of the cardiac cycle can be measured as the distance between either two consecutive $P$ waves or two successive QRS complexes (usually $\mathrm{R}$ waves). For practical reasons, as the peak of the $R$ wave is sharp 

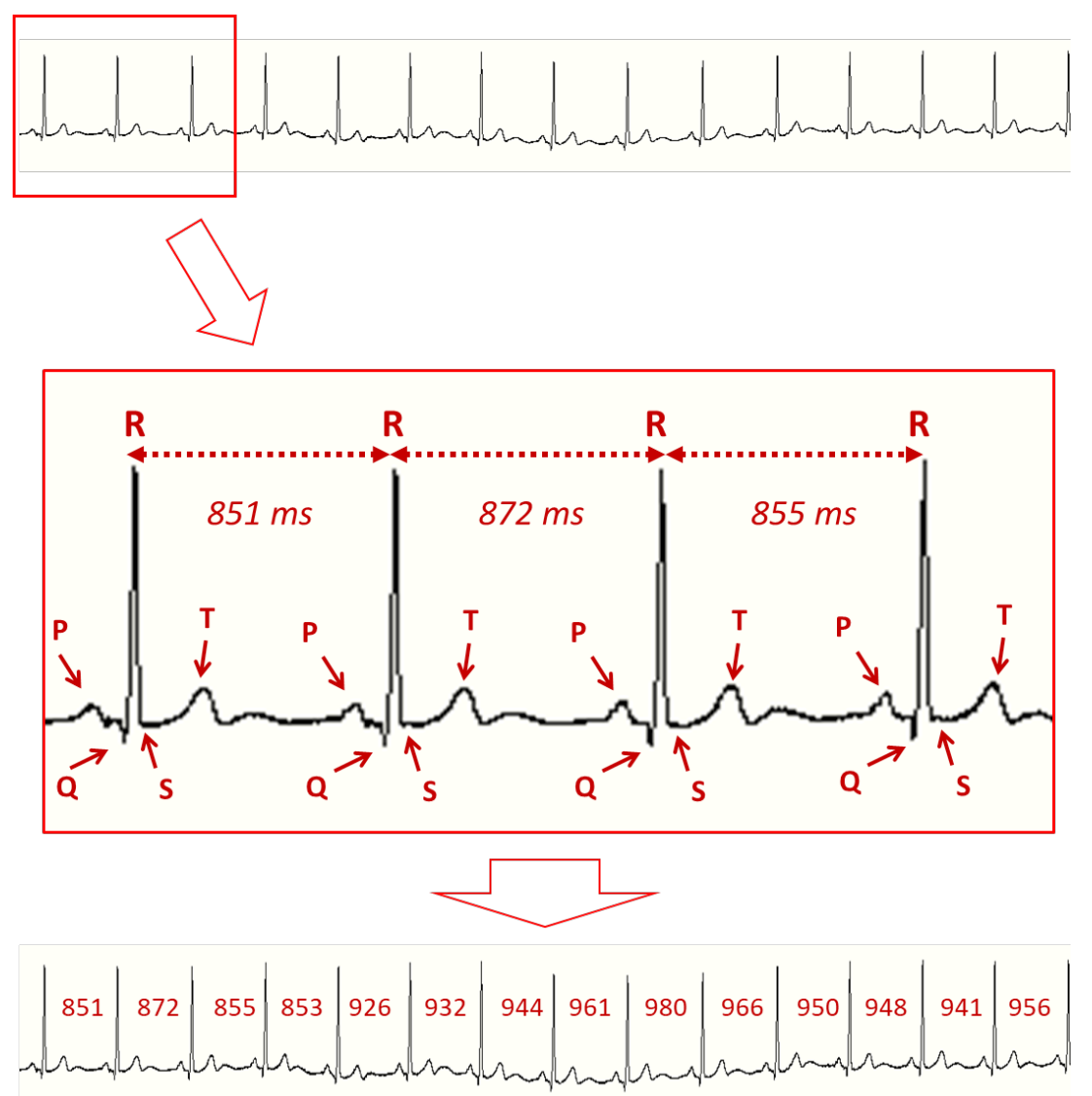

Figure 1. The upper panel shows a strip of normal ECG. The middle panel is zoomed at the first three P-QRS-T waves with the presentation of the distances between the consecutive QRS complexes or peaks of $R$ waves. The measured distances are expressed in milliseconds $(\mathrm{ms})$. The bottom panel shows the same ECG strip with measured RR intervals

and better defined than the front or maximum of a $P$ wave, it is more accurate to measure $R R$, not PP intervals. Therefore, RR intervals, i.e., the distances between consecutive $R$ waves, are the commonly accepted measure of the duration of cardiac cycles $[1,3]$.

Momentary heart rate (HR) is a simple mathematical inverse of the duration of the cardiac cycle or RR interval. Longer RR intervals correspond to a slower $H R$, shorter RR intervals to faster $H R$, with the length of RR intervals or $H R$ changing with each beat. Compared with the previous cardiac cycle, the next cycle can shorten or lengthen, evident as the HR acceleration or deceleration, respectively.

The sinus node is a complex structure composed of many cells, each of which can initiate action potential depolarising other cells within this node, as well as the remaining cardiomyocytes of the whole heart $[1,2,4-11]$. There are many variations in the sinus node shape and size but generally, it is a crescent-like or spindle-shaped structure consisting of head, body and tail, with a maximal length of $14 \mathrm{~mm}$, height up to $8 \mathrm{~mm}$ and width up to $4 \mathrm{~mm}[1,5]$. One to ten radiations of different length and orientation extend from it [5]. Typically, the spindle- and spider-shaped cells, which are smaller, less striated and paler staining, have electrophysiologic features characteristic of pacemaker cells, including spontaneous beating under physiologic conditions. The remaining cells of different histological structure serve to transmit the electrical depolarisation to other cells or as working myocytes and tissue support $[1,2,8,9]$.

Usually, the action potential from the sinus node has a unifocal origin, i.e. a single cell or a group of tightly localised pacemaking cells initiates the depolarisation wave. However, frequently, the action potential comes from multiple pacemaking cells spaced at different parts of the sinus node, which generate multiple wavefronts 
coalescing into one depolarisation wave in 10 to $15 \mathrm{~ms}$. Sometimes, a couple of pacing cells from 2-5 foci located more than $1 \mathrm{~cm}$ apart depolarise asynchronously with short lags 1 to $5 \mathrm{~ms}$ between the activation waves. Regardless of the type, all forms of activation are transferred through discrete sinoatrial exit pathways to the right atrium and the remaining parts of the heart, that is, there is no single leading pacemaker cell, with various types of physiological initiation of the sinus rhythm possible in a healthy human $[1,2,7]$. The initiation and end of the action potential are complicated, depending on several physiological intracardiac and extracardiac factors, for example, the autonomic innervation, oxygen supply, metabolites and hormones [1,3,4,11]. Taking together all these processes, the probability that different cells within the sinus node will generate an action potential of precisely the same duration in two consecutive cardiac cycles is extremely low.

Beat-to-beat change in the duration of $R R$ intervals of sinus origin is a normal physiologi- cal phenomenon $[3,12,13]$. Its typical example is respiratory sinus arrhythmia: HR accelerates during inspiration and decelerates during expiration $[14,15]$. Many other mechanisms than breathing contribute to such instant changes of RR intervals, with the whole phenomenon known as heart rate variability (HRV) $[3,12,13,15]$. The physiology and clinical value of HRV have been extensively studied for over four to five decades and it has earned dedicated guidelines [12,13]. In 2006, our group published the first paper on the existence of another genuine physiological phenomenon related to HRV - heart rate asymmetry (HRA) [16]. Based on studies in healthy individuals, we demonstrated that HRA is a phenomenon caused by the different behaviour of HR accelerations and decelerations, which unequally contribute to the short-, long-term and total HRV as well as the complexity and microstructure of HR [16-24]. Figure 2 presents a local tachogram with consecutive RR intervals with the Poincare plot (one of the methods for HRV analysis) of RR intervals
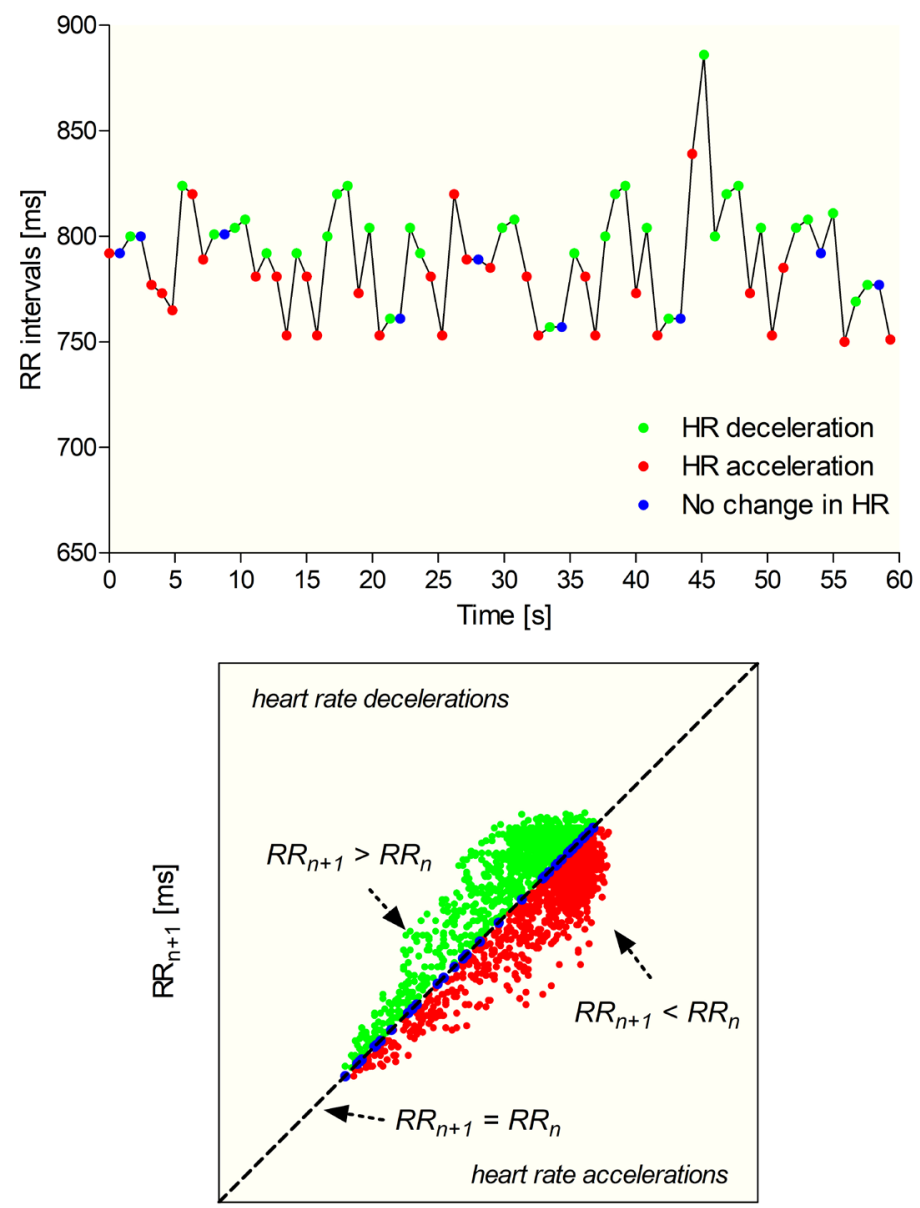

Figure 2. Upper panel shows a local tachogram of consecutive RR intervals from ECG recording of 1-minute duration. RR intervals longer than the previous one are labelled as HR deceleration (green dots), shorter as HR acceleration (red dots), or of the same duration, as no change HR (blue dots). The lower panel shows the Poincare plot of all $R R$ intervals in the space of $\left(R R_{n+1}\right.$, $\mathrm{RR}_{\mathrm{n}}$ ) corresponding to all pairs of two consecutive beats $\left(R R_{n}\right.$ and $\left.R R_{n+1}\right)$. This plot depicts the distribution and relation between such pairs, clearly separating HR decelerations (green dots) from accelerations (red dots). Of note, points described by pairs of RR intervals with precisely the same duration $\left(R R_{n}=R R_{n+1}\right)$ are placed on the identity line (broken diagonal line), represented by blue dots in Figure 2. The Poincare plot of RR intervals in the space $\left(R_{n}, R_{n+1}\right)$ explicitly visualises the existence of HRA

$\mathrm{RR}_{\mathrm{n}}[\mathrm{ms}]$ 
showing the clear separation between HR accelerations and decelerations.

In this review, the concept of the HR microstructure and its asymmetric properties $[20,25]$ will be explained in detail. Additionally, we summarise the clinical findings and show how sampling frequency of ECG can substantially change the number of acceleration, deceleration and neutral runs making up the HR microstructure.

\section{Heart rate microstructure}

Consecutive values of HR, e.g., 75, 76, 74, 72, 78, 80 beats/minute, or RR intervals like $800,789,811$, $833,769,750 \mathrm{~ms}$, create sequences or a series of data points ordered according to their appearance in time, known as the HR time series or RR interval time series, respectively. An RR interval time series is the primary data source for computations of both HRV and HRA. This review will focus only on the RR interval time series.
The order of RR intervals in an ECG of a healthy individual is not random, rather the net result of spontaneous depolarisations of the sinus node modulated by many physiological mechanisms $[1,3,12,13]$. Figure 3 shows two different tachograms of two-minute duration composed from precisely the same RR interval data. In the first case, the order of RR intervals is physiological and comes from an ECG acquired during slow-paced breathing at a rate of 6 breaths/minute, whereas the original order of RR intervals from the same ECG has been destroyed by random shuffling in the second case. The mean value and standard deviation of all RR intervals for both data sets are precisely the same, i.e. $1068 \mathrm{~ms}$ and 115.2 $\mathrm{ms}$, respectively, however, as clearly visible, the microstructure of both RR time series is entirely different. All RR intervals in the dataset with the original order of RR interval follow breathing (12 waves correspond to 12 breathing cycles recorded during 2 minutes), for the shuffled RR intervals there is no clear and repeated pattern.
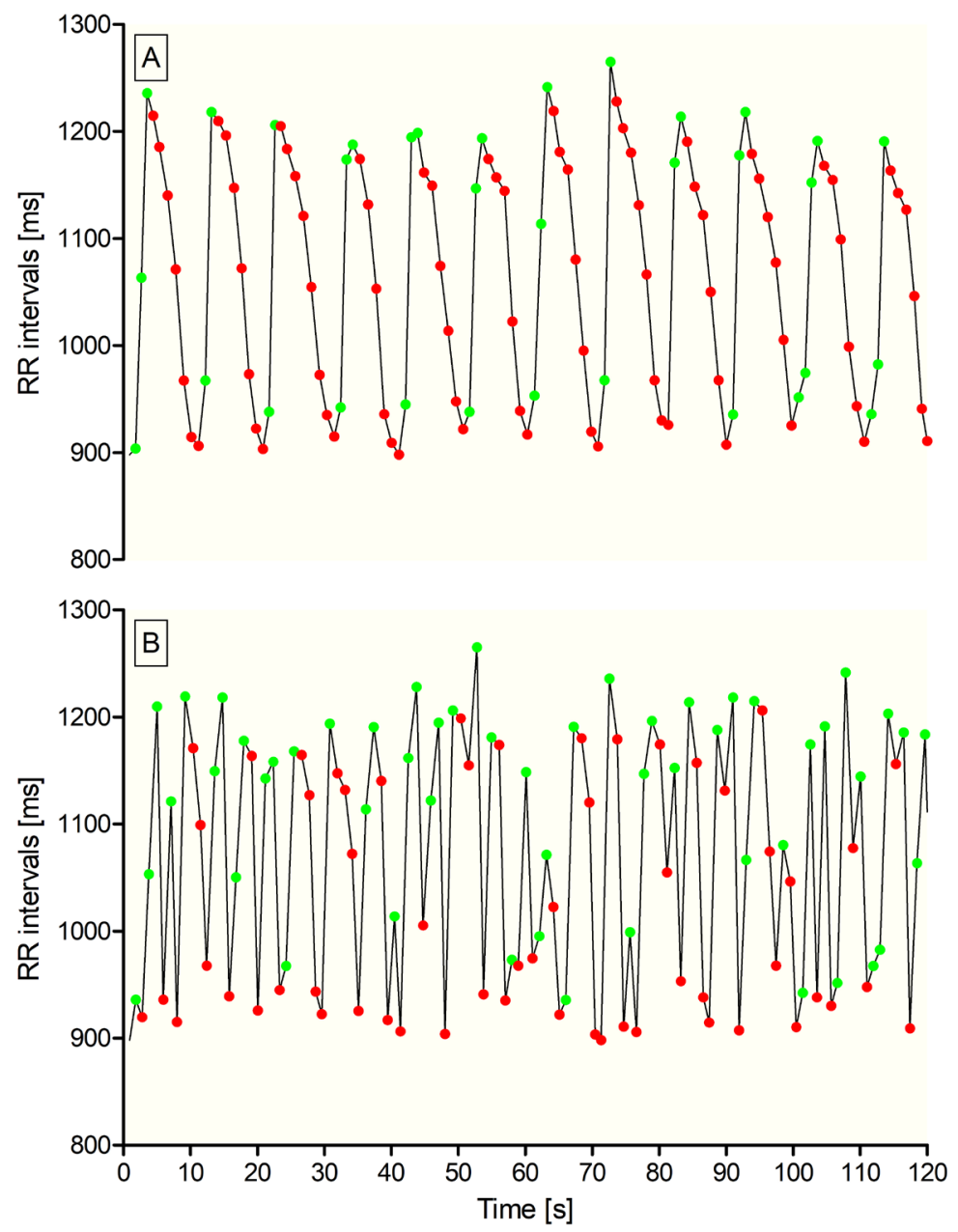

Figure 3. Panel A shows oscillations of RR intervals which are in concordance with the paced breathing at a rate of 6 breaths/minute. This tachogram starts with HR decelerations (green dots) corresponding to expiration, followed by HR accelerations during inspiration, this pattern repeats during each breathing cycle. Panel B shows the same $\mathrm{RR}$ intervals but with completely disrupted physiological order, i.e. all RR intervals are randomly shuffled. The distribution of HR accelerations and decelerations is random, with no repeated pattern 
As evident in Figure 3, for the slow breathing dataset, all RR intervals cluster in groups of either decelerations or accelerations, the socalled monotonic runs. In such a run, consecutive values always change in one direction, either increasing or decreasing. For RR intervals, the monotonic run means that cardiac cycles either prolong for HR decelerations or shorten for HR accelerations. In Figure $\mathbf{3}$, there are runs (or series) of RR intervals of different length, forming two to four consecutive HR decelerations and six to eight successive HR accelerations for the physiological data. In contrast, for RR intervals with the shuffled order, these data are more scattered, with the longest monotonic runs much shorter for both HR accelerations and decelerations. Of note, single HR decelerations and accelerations become more common.

For the analysis of the HR microstructure, all $R R$ intervals are separated into monotonic runs of different length as shown in Table 1 [24-26]. The shortest possible change in RR interval is a single deceleration or acceleration, such singlets are also termed a run, i.e. a deceleration run of length 1 (DR1) or an acceleration run of length 1
(AR1). Two consecutive RR intervals which either increase or decrease their duration are pairs of decelerations (DR2) and accelerations (AR2). Runs of much longer length are naturally occurring, for instance of the length of ten consecutive decelerations (DR10) or twelve accelerations in a row (AR12).

Simple counting statistics are applied to quantify the number of HR acceleration and deceleration runs, the total number of beats in the runs of specific length is counted, then divided by all normal RR intervals [24-26]. The relative contribution of each type of monotonic run to all sinus beats is presented, usually as a percentage or sometimes as a part of 1 [24-26]. For instance, 15 accelerations forming five runs of three consecutive accelerations (AR3) in a recording of 300 sinus RR intervals have a relative contribution of $5 \%$. In another example, in a longer recording composed of 100,000 RR intervals of sinus origin, 6,567 single decelerations (DR1) have a contribution of $6.57 \%, 2,332 \mathrm{RR}$ intervals in the form of deceleration pairs (DR2) are quantified as $2.33 \%$, 492 RR intervals forming triples of decelerations (DR3) correspond to $0.49 \%$ contribution whereas

\begin{tabular}{|c|c|c|c|}
\hline $\begin{array}{l}\text { RR interval } \\
\text { [ms] }\end{array}$ & $\begin{array}{l}\text { Type of HR } \\
\text { change }\end{array}$ & $\begin{array}{c}\text { Number of RR intervals changing } \\
\text { in the same direction }\end{array}$ & $\begin{array}{l}\text { The label of } \\
\text { monotonic run }\end{array}$ \\
\hline 898 & reference value & - & \\
\hline 904 & deceleration & \multirow[t]{2}{*}{2} & \multirow[t]{2}{*}{ DR2 } \\
\hline 954 & deceleration & & \\
\hline 940 & acceleration & 1 & AR1 \\
\hline 948 & deceleration & 1 & DR1 \\
\hline 930 & acceleration & \multirow[t]{3}{*}{3} & \multirow[t]{3}{*}{ AR3 } \\
\hline 918 & acceleration & & \\
\hline 902 & acceleration & & \\
\hline 927 & deceleration & \multirow[t]{6}{*}{6} & \multirow[t]{6}{*}{ DR6 } \\
\hline 933 & deceleration & & \\
\hline 945 & deceleration & & \\
\hline 953 & deceleration & & \\
\hline 960 & deceleration & & \\
\hline 968 & deceleration & & \\
\hline 960 & acceleration & \multirow[t]{2}{*}{2} & \multirow[t]{2}{*}{ AR2 } \\
\hline 942 & acceleration & & \\
\hline 950 & deceleration & 1 & DR1 \\
\hline 948 & acceleration & \multirow[t]{4}{*}{4} & \multirow[t]{4}{*}{ AR4 } \\
\hline 938 & acceleration & & \\
\hline 931 & acceleration & & \\
\hline 925 & acceleration & & \\
\hline 936 & deceleration & 1 & DR1 \\
\hline 920 & acceleration & 1 & AR1 \\
\hline 927 & deceleration & \multirow[t]{2}{*}{2} & \multirow[t]{2}{*}{ DR2 } \\
\hline 941 & deceleration & & \\
\hline
\end{tabular}

Table 1. An example of proper identification of each RR interval as HR deceleration or acceleration. The identification process is simple and based on the comparison of duration of each specific RR interval with the previous RR interval. The number of RR intervals changing in the same direction (either prolonging for HR decelerations or shortening for accelerations) is counted, with specific monotonic runs labelled based on the number of RR intervals changing in the same direction 
$84 \mathrm{RR}$ intervals found as runs of four decelerations contribute to $0.08 \%$ of all sinus beats. The same approach of counting statistics is also applied for reporting the contribution of premature ventricular (or supraventricular) beats to all recorded beats in the longer Holter ECG recordings, for example of 24-hour duration. In such reports, the relative number of ventricular beats is shown, for example as $0.5 \%$ or $19 \%$.

In 2011, we showed that the HR microstructure had asymmetric properties in our physiological study of eighty-seven ECG recordings of up to 24-hour duration acquired from healthy people [24]. The number of acceleration runs was usually significantly higher (with the exceptions of AR3s vs DR3s and AR4s vs DR4s) than deceleration runs. Additionally, the longest runs in the same people came from 24 accelerations (AR24) and only 19 decelerations (DR19), with the average longest runs formed of over 15 accelerations and over 12 for decelerations.

Table 2 [25] summarises the relative contributions of RR intervals creating acceleration and deceleration runs to the total number of sinus beats in the 24-hour ECG recordings from healthy people. It is visible that, except for monotonic runs of the length 3 and 4, in the majority of other lengths, acceleration runs have a significantly larger contribution to all sinus beats in these recordings. For monotonic runs of the length 3 , there were more DRs than ARs, while the number of monotonic runs of the length 4 was comparable both for decelerations and accelerations.

To check whether the asymmetric properties of the monotonic runs method were not artificially generated, we compared the distribution of the acceleration and deceleration runs after shuffling the original order of RR intervals into a random one in each of the 87 recordings [24]. For the shuffled data, there were no significant differences between acceleration and deceleration runs of any length. Additionally, the longest monotonic runs were composed of nine consecutive beats (AR9 and DR9), and the averaged longest runs were length seven both for decelerations and accelerations (AR7 and DR7). Interestingly, there were significantly fewer runs which were shorter and more runs which were longer for RR interval time series with the original order than after its disruption by random shuffling. Thus, we concluded that the input of HR accelerations and decelerations to the distribution of monotonic runs is unequal, hence asymmetric, that the distribution of acceleration runs and deceleration runs differs between physiological and shuffled data, with some not completely understood physiological mechanisms creating longer monotonic runs at the cost of shorter runs (i.e., singlets and pairs of HR accelerations and decelerations) [24].

Also, in 2011, we published, in a co-operation with the group of Professor Georg Schmidt from Munich in Germany, the first clinical study on the HR microstructure [26]. We analysed the predictive value of $\mathrm{HR}$ deceleration runs measured in the 24-hour ECGs from 1455 (training sample) and 946 (validation sample) survivors of myocardial infarction. Patients at high risk of death in the long-term follow-up of median duration of 24 months had significantly more single decelerations (DR1) and fewer deceleration runs of the length between 2 and 10 (DR2-DR10) than the low-risk individuals. The reduced number of

\begin{tabular}{lccccc}
\multirow{2}{*}{$\begin{array}{c}\text { Runs of } \\
\text { the length }\end{array}$} & \multicolumn{2}{c}{ Deceleration runs [\%] } & \multicolumn{2}{c}{ Acceleration runs [\%] } & P-value \\
\cline { 2 - 5 } & Median & IQR & Median & IQR & \\
\hline 1 & 12.64 & 5.60 & 13.18 & 5.03 & 0.003 \\
\hline 2 & 13.71 & 4.89 & 14.63 & 3.54 & $<0.001$ \\
\hline 3 & 8.71 & 4.16 & 7.16 & 2.59 & $<0.001$ \\
\hline 4 & 3.54 & 1.98 & 3.55 & 1.33 & n.s. \\
\hline 5 & 1.82 & 1.28 & 2.32 & 1.33 & $<0.001$ \\
\hline 6 & 1.01 & 0.94 & 1.60 & 1.29 & $<0.001$ \\
\hline 7 & 0.49 & 0.53 & 0.95 & 0.91 & $<0.001$ \\
\hline 8 & 0.23 & 0.25 & 0.56 & 0.61 & $<0.001$ \\
\hline 9 & 0.1 & 0.12 & 0.32 & 0.38 & $<0.001$ \\
\hline 10 & 0.04 & 0.06 & 0.16 & 0.21 & $<0.001$ \\
\hline 11 & 0.02 & 0.04 & 0.08 & 0.1 & $<0.001$ \\
\hline 12 & 0.01 & 0.02 & 0.05 & 0.08 & $<0.001$ \\
\hline
\end{tabular}

Table 2. Comparison of the relative contributions of acceleration runs and deceleration runs of different length to the total number of sinus beats in the 24-hour ECG recordings from healthy people. The statistical comparison was made with the nonparametric Mann-Whitney test. (IQR - interquartile range) [25] 
DR4 up to $0.05 \%$ was the best single predictor of three separate modes of premature death, i.e., all-cause mortality, cardiac death, and sudden cardiac death both in the training and validations samples, whereas patients with a preserved number of DR2 $>5.4 \%$, DR4 $>0.05 \%$, and DR8 $>0.005 \%$ belonged to the low-risk group of all observed death-related clinical outcomes.

These relative values require some additional comment. If in a 24-hour ECG of a myocardial infarction survivor there are 100,000 sinus beats of which more than $5,400 \mathrm{HR}$ decelerations are in DR2s, more than 50 in DR4s and more than five in DR8, then such a person is at low risk of premature death, whereas if there are 50 or fewer RR intervals which form DR4, then the patient is at an increased risk. Although not presented in that paper in the form of numbers, similar findings were made for HR acceleration runs and commented on in the discussion. The reduced relative numbers of AR2-AR10 were found in highrisk patients, although the prognostic value of acceleration runs was lower in comparison to the deceleration runs.

In general, the distribution of deceleration and acceleration runs in survivors of myocardial infarction who were at an increased risk of premature death converged to the distribution of the shuffled RR intervals as in our physiological study with 24-hour ECGs from healthy individuals. In other words, mechanisms responsible for forming HR accelerations and decelerations into longer runs were preserved in low-risk and attenuated in high-risk post-infarction patients.

We also tested, in co-operation with Professor Tuomo Nieminen from Oulu in Finland, the predictive value of single accelerations and decelerations in pre-exercise ECGs of at least 1-minute duration collected in 944 consecutive patients from the Finnish Cardiovascular Study [27]. All patients had clinical indications for the treadmill exercise test, after which they were followed for a mean of nearly 57 months. Those with increased relative numbers of $A R 1>16.85 \%$ or $D R 1>17.7 \%$ had a significantly higher total mortality than other patients. Increased numbers of AR 1 and DR1 were also associated with a higher risk for cardiovascular death and sudden cardiac death. As the mean duration of pre-exercise ECGs was short, between 1 minute and a couple of minutes, studying the predictive value of longer runs was impossible for statistical reasons (too low statistical power). However, there was an essential similarity to the study in post-infarction patients - high-risk patients in these two different groups had an increased number of AR1s and DR1s. These findings again highlight that in the highrisk cardiac patients, the numbers of AR1 and DR1 increased as in the case of the shuffled data from healthy people.

Billois et al. compared the asymmetric properties of HR microstructure in 32 premature infants, 16 of whom were infected and at risk of sepsis [28]. The study was performed with the use of 30-minute ECGs, and the authors analysed the absolute numbers of HR decelerations. Significant differences were observed between infants with and without infection in the number of deceleration but not acceleration runs. Infants at an increased risk of sepsis presented fewer DR1s and more DR3s and DR4s. The normal heart rate in healthy infants is always faster than in older children and adults [29]. Bradycardia in infants may be secondary to several causes, e.g. hypoxemia, toxaemia or sepsis [30]. Billois et al. showed for the first time that infants at risk of sepsis express a significant change in the HR microstructure in the form of an increased number of HR decelerations, which appear to group into longer runs at the cost of deceleration singlets [28].

Using the 300-minute ECGs recorded during sleep from seventy-eight patients with suspected obstructive sleep apnoea (OSA), in co-operation with Professor Adrian Baranchuk from Kingston, ON, Canada, we investigated the relationship between the severity of this disease and the asymmetric features of HR microstructure [31]. Patients with severe OSA had fewer DR1s and AR1s than subjects with moderate OSA, and more AR5, AR10s and DR5s, DR8s than patients with no or mild OSA. Additionally, the longest acceleration runs were significantly longer in patients from the severe OSA (on the average of the length 9) than in individuals with no or a mild form of this disease (on average of the length 8). Interestingly, the severity of OSA was not significantly associated with the values of any measures of HRV (including spectral analysis).

The first impression from the OSA study was that it contrasts with the findings from post-infarction patients $[26,31]$. While high-risk survi- 
vors of myocardial infarction had fewer longer runs in the 24-hour ECGs [26], the most severe OSA patients had more such runs in the 300 minute ECG recorded during sleep [31]. Regardless of technical differences, duration of the recording, comparing sleep ECGs vs the whole 24-hour ECGs, one important feature must be underlined, OSA patients, particularly with the more severe form, have many repeated episodes of apnoea and hypopnea during sleep [32,33]. The hypopnea/apnoea episode lasts at least 10 seconds, and patients with severe OSA have at least 30 such incidents during the polysomnographic recording (ECG is one of several vital signals recorded during polysomnography) [33]. In the studied patients, the average number of hypopnea/apnoea episodes was 43 [31]. In OSA patients, after each hypopnea/apnoea, there was increased breathing which, for a short period, was deeper and faster. Typically during hypopnea or apnoea, there is a reduction in $\mathrm{HR}$, bradycardia is common, and even short pauses may appear, when breathing is restored, HR rapidly increases and such erratic breathing strongly influences HR [31,33]. It seems that more episodes of hypopnea or apnoea triggered more HR decelerations, whereas during the consequent increase in breathing more HR accelerations were found. Both HR decelerations and accelerations clustered in longer runs more commonly in patients with more severe OSA [31].

In 2017, Jiang et al. published their study on the $H R$ accelerations and decelerations in a larger group of 231 OSA patients and analysed 6-hour ECGs [34]. They confirmed our observations that individuals with severe OSA had more longer HR acceleration runs (AR2-AR5, and undefined long $A R$ ) and deceleration runs (DR5 and undefined long DR). Additionally, they found in a prospective substudy of 39 OSA (8 moderate and 31 severe) patients that a one-night treatment with a continuous positive airway pressure reduced the number of longer ARs and DRs. It was the first-ever observation from a prospective clinical study proving that a planned medical intervention (in this case, the continuous positive airway pressure applied for one night) significantly modified the HR microstructure.

Recently, Kong et al. studied 151 patients with chronic obstructive pulmonary disease (COPD) and 45 patients without COPD [35], reporting that
COPD patients had significantly fewer DR2s, DR4s and DR8s than the remaining individuals. Additionally, these authors reported that the numbers of longer DRs were negatively associated with the presence of arrhythmia, i.e. premature atrial beats, supraventricular tachycardias, and premature ventricular beats, that is, reduced numbers of DR2, DR4 and DR8 were associated with a higher risk of cardiac arrhythmias. Unfortunately, there are no data on the deceleration runs of other length or any acceleration runs. However, this study is clinically relevant as it shows the predictive value of the analysis of the HR microstructure and its asymmetry in another group of patients, this time with COPD.

\section{Limitations of the analy- sis of HR microstructure}

The main limitation of counting the number of monotonic runs is the length of the recording, as the probability of occurrence of longer runs is low in short recordings lasting only a couple of minutes. For this reason, longer runs, particularly very long runs of the length of over 12 and more consecutive RR intervals changing in the same direction are easier to spot in ECG recordings of several hours duration $[20,25]$. Thus, for ECG recordings lasting up to 5 or 10 minutes, it is better to limit the analysis of HR microstructure to shorter runs up to 4 beats. For ECGs of moderate length such as up to 30-60-minute recordings, the analysis of the monotonic runs should be limited to 8 beats. For the 24-hour ECGs, it is common to find longer monotonic runs between 12 and 16 beats, even of the length of 24 beats $[20,25]$. As far as we know, no data exist with the HR microstructure analysis in ECG recordings extending for more than 24 hours.

Another limitation is the sampling frequency of the recorded ECG. Previous studies on the microstructure of HRA used a lower sampling frequency of $200 \mathrm{~Hz}$ for the 24-hour Holter ECGs, which translates into the precision of RR intervals of $5 \mathrm{~ms}[20,25]$. Consequently, there was a substantial number of so-called neutral runs (up to $6-7 \%$ of all beats), i.e., such consecutive RR intervals which have identical duration. For instance, the neutral run of three (NR3) is composed of four successive and equal RR intervals with the first as 
the reference for the $2^{\text {nd }}$, the $2^{\text {nd }}$ for the $3^{\text {rd }}$, and the $3^{\text {rd }}$ for the $4^{\text {th }} \mathrm{RR}$ interval. RR intervals before the first and the fourth RR intervals must be different. In total, the number of comparisons showing no change for this particular neutral run equals three. The following time series of RR intervals is more explanatory: $1000 \mathrm{~ms}$ ( $1^{\text {st }} \mathrm{RR}$ interval), 1000 $\mathrm{ms}\left(2^{\text {nd }} \mathrm{RR}\right.$ interval), $1000 \mathrm{~ms}$ ( $3^{\text {rd }} \mathrm{RR}$ interval), and $1000 \mathrm{~ms}$ ( $4^{\text {th }} \mathrm{RR}$ interval) (Figure 4).
In the study of post-infarction patients, the sampling frequency of the 24-hour ECGs was even lower, $128 \mathrm{~Hz}$ [26]. The same sampling frequency was applied ECGs from OSA patients [31]. However, none of these studies revealed the number of neutral runs. The information on the sampling frequency and the number of neutral runs should be reported in all studies on the HR microstructure.

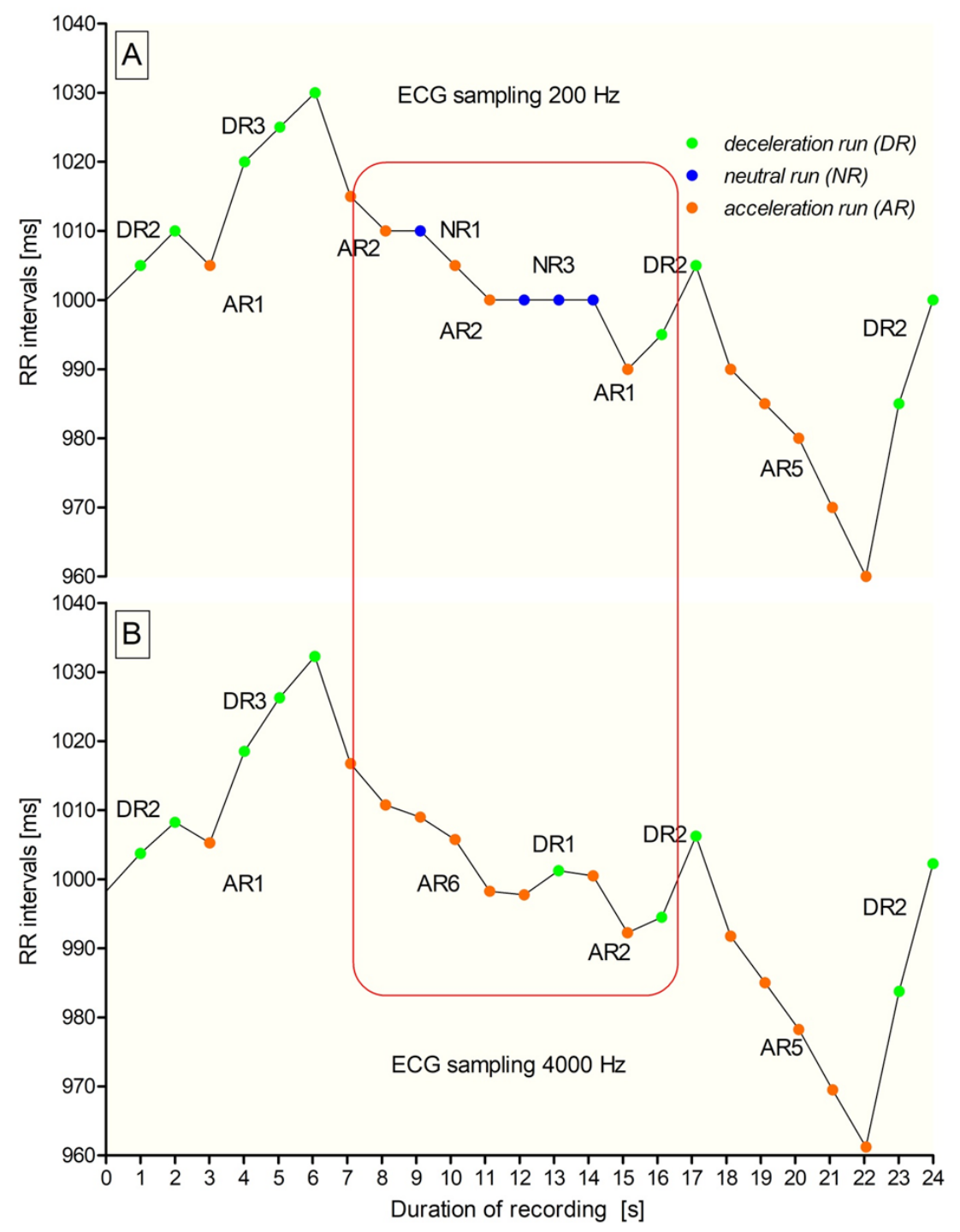

\begin{tabular}{|c|c|c|c|c|c|c|c|c|c|c|}
\hline \multirow{2}{*}{$\begin{array}{c}200 \mathrm{~Hz} \text { ECG } \\
\text { sampling }\end{array}$} & 1015 & 1010 & 1010 & 1005 & 1000 & 1000 & 1000 & 1000 & 990 & 995 \\
\hline & & A & $N$ & A & A & $N$ & $N$ & $N$ & A & D \\
\hline & & & & & & & & & & \\
\hline \multirow{2}{*}{$\begin{array}{c}4000 \mathrm{~Hz} \text { ECG } \\
\text { sampling }\end{array}$} & 1016,75 & 1010,75 & 1009,00 & 1005,75 & 998,25 & 997,75 & 1001,25 & 1000,50 & 992,25 & 994,50 \\
\hline & & A & A & A & A & A & D & A & A & D \\
\hline
\end{tabular}

Figure 4. Effect of sampling frequency on the measurement of $\mathrm{RR}$ intervals and their labelling. The upper panel shows a local tachogram for the ECG recorded at the sampling frequency of 200 $\mathrm{Hz}$ whereas the lower panel presents the same ECG recorded at the sampling frequency of 4000 $\mathrm{Hz}$. In the upper panel, there are neighbouring RR intervals of identical duration (marked with blue dots), so-called neutral runs (NR), and two examples of such runs are presented as NR1 and NR3. The lower panel, however, shows that these RR intervals did not have the same duration, and instead of NRs, there were some HR accelerations (ARs) and decelerations (DRs). A higher sampling frequency of the recorded ECG limits the number of falsely identified NR, providing a proper number of acceleration and deceleration runs. The bottom panel shows the values of $\mathrm{RR}$ intervals with the corresponding labelling for both types of ECG sampling 
Newer long-term recorders have a much higher sampling frequency of ECG between $1000 \mathrm{~Hz}$ to $4000 \mathrm{~Hz}$, which may translate to the identification of RR intervals with the precision between 1 to $0.25 \mathrm{~ms}$. However, due to the intrinsic properties of the ECG signal, such high frequency might increase the signal-to-noise ratio. Additionally, at higher sampling frequencies, the peak of $\mathrm{R}$ wave flattens and no longer resembles a point. Taken together, an increase in the noise and flattening of the peaks of $R$ waves might interfere with the proper measurement of the distances of RR intervals. Although it is tempting to believe that higher sampling frequencies might improve the precision of RR intervals measurement and limit or even zero the number of neutral runs, this is uncertain and requires separate studies. However, the currently applied devices record ECG at a much lower sampling frequency between 125 and 250 $\mathrm{Hz}$, so there is room for the improvement of $\mathrm{RR}$ intervals measurement. In effect, the analysis of the asymmetric properties of HR microstructure should be more accurate and increase the number of adequately detected longer DRs and ARs. As discussed in a previous study [20], the neutral runs seem to be an artificial effect of low sampling frequency than a genuine physiological phenomenon. Studying the distribution of acceleration and deceleration runs in Holter ECGs recorded at a much higher frequency than $125-250 \mathrm{~Hz}$ [20] might result in a lower number of or even no neutral runs, and more precise description of the asymmetric features of HR microstructure.

In summary, the analysis of the HR microstructure and its asymmetric properties seems to be attractive from both a physiological and clinical point of view. It helps to visualise how the RR time series are distributed and to understand how HRV is created. Studying the HR microstructure in clinical scenarios has been demonstrated not only to have predictive value in certain groups of patients but also to monitor the effects of some interventions (e.g., the application of continuous positive airway pressure in OSA patients). In contrast to many other methods transferred to HRV from different scientific areas like astronomy, economy or physics, the monotonic runs methods were deliberately developed to study specific features of normal heart rate and further explore HRA $[12,13,15]$. We believe that this method deserves more attention and requires further investigation.
Acknowledgements

\section{Conflict of interest statement}

The authors declare no conflict of interest.

\section{Funding sources}

There are no sources of funding to declare.

\section{References}

1. Tomaselli GF, Rubart M, Zipes DP. Mechanisms of Cardiac Arrhythmias. In: Braunwald's Heart Disease: A Textbook of Cardiovascular Medicine. 10th Edition. Saunders; 2014:619-49.

2. Fedorov VV, Glukhov AV, Chang R, Kostecki G, Aferol H, Hucker WJ, Wuskell JP, Loew LM, Schuessler RB, Moazami N, Efımov IR. Optical Mapping of the Isolated Coronary-Perfused Human Sinus Node. Journal of the American College of Cardiology. 2010 Oct;56(17):1386-1394. https://doi.org/10.1016/j. jacc. 2010.03.098

3. Shaffer F, McCraty R, Zerr CL. A healthy heart is not a metronome: an integrative review of the heart's anatomy and heart rate variability. Frontiers in Psychology. 2014 Sep 30;5. https://doi.org/10.3389/ fpsyg.2014.01040 PMID 25324790

4. Opthof T. The normal range and determinants of the intrinsic heart rate in man. Cardiovascular Research. 2000 Jan 1;45(1):177-184. https://doi.org/10.1016/ s0008-6363(99)00322-3

5. Sanchez-Quintana D. Sinus node revisited in the era of electroanatomical mapping and catheter ablation. Heart. 2005 Feb 1;91(2):189-194. https://doi. org/10.1136/hrt.2003.031542

6. Moïse NS, Flanders WH, Pariaut R. Beat-to-Beat Patterning of Sinus Rhythm Reveals Non-linear Rhythm in the Dog Compared to the Human. Frontiers in Physiology. 2020 Jan 22;10. https://doi.org/10.3389/ fphys.2019.01548 PMID 32038271

7. Boineau JP, Canavan TE, Schuessler RB, Cain ME, Corr PB, Cox JL. Demonstration of a widely distributed atrial pacemaker complex in the human heart.. Circulation. 1988 Jun;77(6):1221-1237. https://doi. org/10.1161/01.cir.77.6.1221

8. Ho SY, Sánchez-Quintana D. Anatomy and pathology of the sinus node. Journal of Interventional Cardiac Electrophysiology. 2015 Aug 30;46(1):3-8. https:// doi.org/10.1007/s10840-015-0049-6

9. Peters $\mathrm{CH}$, Sharpe EJ, Proenza C. Cardiac Pacemaker Activity and Aging. Annual Review of Physiology. 2020 Feb 10;82(1):21-43. https://doi.org/10.1146/ annurev-physiol-021119-034453

10. Boyett $M$. The sinoatrial node, a heterogeneous pacemaker structure. Cardiovascular Research. 2000 Sep;47(4):658-687. https://doi.org/10.1016/s00086363(00)00135-8

11. John RM, Kumar S. Sinus Node and Atrial Arrhythmias. Circulation. 2016 May 10;133(19):1892-1900. https://doi.org/10.1161/circulationaha.116.018011

12. Electrophysiology TFOTES. Heart Rate Variability. Circulation. 1996 Mar;93(5):1043-1065. https://doi. org/10.1161/01.cir.93.5.1043 
13. Sassi R, Cerutti S, Lombardi F, Malik M, Huikuri HV, Peng C, Schmidt G, Yamamoto Y, Gorenek B, Lip GY, Grassi G, Kudaiberdieva G, Fisher JP, Zabel M, Macfadyen $R$. Advances in heart rate variability signal analysis: joint position statement by the e-Cardiology ESC Working Group and the European Heart Rhythm Association co-endorsed by the Asia Pacific Heart Rhythm Society. Europace. 2015 Jul 14;17(9):13411353. https://doi.org/10.1093/europace/euv015

14. Berntson GG, Cacioppo JT, Quigley KS. Respiratory sinus arrhythmia: Autonomic origins, physiological mechanisms, and psychophysiological implications. Psychophysiology. 1993 Mar;30(2):183-196. https:// doi.org/10.1111/j.1469-8986.1993.tb01731.x

15. Piskorski J, Guzik P, Krauze T, Żurek S. Cardiopulmonary resonance at $0.1 \mathrm{~Hz}$ demonstrated by averaged Lomb-Scargle periodogram. Open Physics. 2010 Jan 1;8(3). https://doi.org/10.2478/s11534-009-0101-1

16. Guzik P, Piskorski J, Krauze T, Wykretowicz A, Wysocki $\mathrm{H}$. Heart rate asymmetry by Poincaré plots of RR intervals. Biomedizinische Technik/Biomedical Engineering. 2006 Oct;51(4):272-275. https://doi. org/10.1515/bmt.2006.054

17. Piskorski J, Guzik P. Geometry of the Poincare plot ofRRintervals and its asymmetry in healthy adults. Physiological Measurement. 2007 Feb 19;28(3):287300. https://doi.org/10.1088/0967-3334/28/3/005

18. Guzik P, Piskorski J, Krauze T, Wykretowicz A, Wysocki $\mathrm{H}$. Partitioning total heart rate variability. International Journal of Cardiology. 2010 Sep;144(1):138139. https://doi.org/10.1016/j.ijcard.2008.12.151

19. Piskorski J, Guzik P. Asymmetric properties of long-term and total heart rate variability. Medical \& Biological Engineering \& Computing. 2011 Sep 28;49(11):1289-1297. https://doi.org/10.1007/s11517011-0834-z

20. Piskorski J, Guzik P. The structure of heart rate asymmetry: deceleration and acceleration runs. Physiological Measurement. 2011 Jun 7;32(8):1011-1023. https://doi.org/10.1088/0967-3334/32/8/002

21. Piskorski J, Guzik P. Compensatory properties of heart rate asymmetry. Journal of Electrocardiology. 2012 May;45(3):220-224. https://doi.org/10.1016/j. jelectrocard.2012.02.001

22. Mieszkowski D, Kośmider M, Krauze T, Guzik P, Piskorski J. Asymmetric detrended fluctuation analysis reveals asymmetry in the RR intervals time series. Journal of Applied Mathematics and Computational Mechanics. 2016 Mar;15(1):99-106. https://doi. org/10.17512/jamcm.2016.1.10

23. Piskorski J, Kosmider M, Mieszkowski D, Krauze T, Wykretowicz A, Guzik P. Properties of Asymmetric Detrended Fluctuation Analysis in the time series of RR intervals. Physica A: Statistical Mechanics and its Applications. 2018 Feb;491:347-360. https://doi. org/10.1016/j.physa.2017.09.057

24. Piskorski J, Ellert J, Krauze T, Grabowski W, Wykretowicz A, Guzik P. Testing heart rate asymmetry in long, nonstationary 24 hour RR-interval time series. Physiological Measurement. 2019 Oct 30;40(10):105001. https://doi.org/10.1088/1361-6579/ab42d5
25. Piskorski J. Struktura asymetrii rytmu serca (Structure of heart rate asymmetry). Poznań: Wydawnictwo Naukowe; 2011.

26. Guzik P, Piskorski J, Barthel P, Bauer A, Müller A, Junk $\mathrm{N}$, Ulm K, Malik M, Schmidt G. Heart rate deceleration runs for postinfarction risk prediction. Journal of Electrocardiology. 2012 Jan;45(1):70-76. https://doi. org/10.1016/j.jelectrocard.2011.08.006

27. Guzik P, Nieminen T, Piskorski J, Kaiser W, Viik $\mathrm{J}$, Nikus $\mathrm{K}$, Lehtinen R, Lehtimäki $\mathrm{T}$, Kähönen $\mathrm{M}$. Increased rate of fast-changing microstructure of heart rate asymmetry predicts mortality in patients undergoing a clinically indicated exercise test. Cardiol. J. 2011;18:593.

28. Billois R, Porée F, Beuchée A, Carrault G. Interest of RR deceleration for diagnosis of late onset sepsis. In: IEEE Computing in Cardiology. Kraków; 2012:633-6.

29. Fleming $S$, Thompson $M$, Stevens $R$, Heneghan $C$, Plüddemann A, Maconochie I, Tarassenko L, Mant D. Normal ranges of heart rate and respiratory rate in children from birth to 18 years of age: a systematic review of observational studies. The Lancet. 2011 Mar;377(9770):1011-1018. https://doi.org/10.1016/ s0140-6736(10)62226-x PMID 21411136

30. Baruteau A, Perry JC, Sanatani S, Horie M, Dubin AM. Evaluation and management of bradycardia in neonates and children. European Journal of Pediatrics. 2016 Jan 16;175(2):151-161. https://doi. org/10.1007/s00431-015-2689-z

31. Guzik P, Piskorski J, Awan K, Krauze T, Fitzpatrick M, Baranchuk A. Obstructive sleep apnea and heart rate asymmetry microstructure during sleep. Clinical Autonomic Research. 2013 Jan 24;23(2):91-100. https://doi.org/10.1007/s10286-013-0188-8

32. Bibbins-Domingo K, Grossman DC, Curry SJ, Davidson KW, Epling JW, García FAR, Herzstein J, Kemper $A R$, Krist $A H$, Kurth $A E$, Landefeld $C S$, Mangione CM, Phillips WR, Phipps MG, Pignone MP, Silverstein M, Tseng C. Screening for Obstructive Sleep Apnea in Adults. JAMA. 2017 Jan 24;317(4):407. https://doi. org/10.1001/jama.2016.20325

33. Parati G, Lombardi C, Hedner J, Bonsignore MR, Grote L, Tkacova R, Levy P, Riha R, Bassetti C, Narkiewicz K, Mancia G, McNicholas WT. Position paper on the management of patients with obstructive sleep apnea and hypertension. Journal of Hypertension. 2012 Apr;30(4):633-646. https://doi.org/10.1097/ hjh.0b013e328350e53b

34. Jiang J, Chen X, Zhang C, Wang G, Fang J, Ma J, Zhang $J$. Heart rate acceleration runs and deceleration runs in patients with obstructive sleep apnea syndrome. Sleep and Breathing. 2016 Nov 23;21(2):443-451. https://doi.org/10.1007/s11325-016-1437-6

35. Kong Z, Wang X, Shen S, Liu H, Zhou L, Chen B, Chen $Z$, Yin S. Risk Prediction for Arrhythmias by Heart Rate Deceleration Runs in Patients with Chronic Obstructive Pulmonary Disease. International Journal of Chronic Obstructive Pulmonary Disease. 2020 Mar;Volume 15:585-593. https://doi.org/10.2147/ copd.s234470 